I.

ON CERTAIN POINTS

IN THE

ANATOMY AND PATHOLOGY

of

\title{
BRIGHT'S DISEASE OF THE KIDNEY.
}

II.

ON THE INFLUENCE

OF THE

MINUTE BLOOD-VESSELS UPON THE CIRCULATION.

BY

G E O R G E J O H N O N, M. D., PHYSICIAN TO KING's COLLEGE hospital.

Received Nov. 17th.-Read Dec. 10th, 1867.

Iт is now generally admitted by pathologists and practical physicians that cases of chronic Bright's disease may be arranged in two distinct classes. The first class includes cases in which the kidneys after death are found of small size, of red colour, and with an uneven granular surface; while in the second class are included those cases in which the kidneys are large, white, and smooth on the surface. In a paper which was published in the 42nd vol. of the 'MedicoChirurgical Transactions' I pointed out the chief features in the anatomical characters and in the clinical history of the two classes of cases by which they may be distinguished from each other. 
Although there is now a very general agreement as to the doctrine that a small granular kidney is essentially different from the large, white, smooth kidney, yet there exists some diversity of opinion as to the minute anatomy and pathology of the kidney in the various morbid conditions of the organ which are included under the head of chronic Bright's disease, and I desire now to correct what I conceive to be the error of some doctrines which have recently been promulgated.

Some pathologists maintain that the small granular kidney is the result of an intertubular deposit. I believe this to be an error, and for the following reasons. Those who advocate this doctrine give no sufficient proof of an intertubular deposit, and they take little or no account of those remarkable intratubular changes which have been described and demonstrated by others. ${ }^{1}$ In my own numerous examinations of small granular kidneys I have found no evidence of an intertubular deposit, but, on the contrary, in the wide spaces which commonly exist between the tubes I have seen only the atrophied remains of tubes and capillaries, with occasionally a doubtful appearance of thickening of the matrix. Perhaps the most conclusive proof of the absence of an intertubular deposit is to be found in the fact that these small granular kidneys retain their red colour and their vascularity even when the disease has reached a very advanced stage. This form of diseased kidney is commonly called the "red granular kidney," in contradistinction to the "large white kidney." Now, this red colour of the kidney is utterly inconsistent with the doctrine of an intertubular deposit. Any deposit between the tubes must of necessity obliterate the intertubular capillaries, and the seat of the deposit consequently become pale and exsanguine. In proof of this statement I need only refer to a form of disease in which the intertubular capillaries are filled by an intertubular deposit, namely, the so-called "fibrinous deposits" in the kidney. In the 9th volume of the "Pathological Transactions' I have shown that these so-called

1 See two papers, one by the author and one by Mr. Simon, in the 30th vol. of the 'Medico-Chirurgical Transactions.' 
fibrinous deposits originate in the formation of coagula in the intertubular capillaries. In consequence of this obstruction there is an exudation from the overgorged Malpighian capillaries into the tubes, and the result is the formation of a firm, elevated, circumscribed patch, which is quite pale and anæmic, and which subsequently softens and becomes absorbed, leaving a cicatrix on the surface of the kidney.

The contrast between these anæmic patches, where an intertubular deposit can be proved to exist, and the red, vascular, cortical tissue of a small granular kidney, where an intertubular deposit has been supposed to occur, is very striking, and would of itself be sufficient to disprove the doctrine in question.

It has been said that the disintegration of the glandular epithelium of the convoluted tubes, which $I$ have described as the primary and most constant phenomenon in the small granular kidney, is the result of post-mortem changes. This statement, however, is entirely disproved by the fact that "granular casts," composed of epithelium more or less disintegrated, are almost constantly present in the urine, and afford conclusive evidence that the disintegration of the gland-cells is in continual progress during the lifetime of the patient. The appearance of these granular casts, their number and their size, form important elements in our means of diagnosis and prognosis in this class of cases.

Another doctrine which I believe to be erroneous was first published by Virchow; I allude to the doctrine that the structural changes in the so-called waxy or amyloid degeneration of the kidney are the result of a primary degeneration of the minute arteries and the Malpighian capillaries. This brings me to the main object of this communication, which is to explain the remarkable changes which the minute blood-vessels undergo during the progress of chronic Bright's disease. My explanation of these changes will lead me to correct what I conceive to be the error of Virchow's doctrine.

In a paper which was published in the 33rd volume of the 'Medico-Chirurgical Transactions,' I first described a re- 
markable condition of the minute blood-vessels of the kidney which I had observed in cases of chronic Bright's disease, namely, hypertrophy of the muscular coats of the minute arteries, and thickening of the walls of the Malpighian capillaries while the intertubular capillaries and veins present no appearance of thickening. Since the publication of that paper I have examined numerous specimens of diseased kidneys, and the result has been to confirm, in nearly every particular, the anatomical accuracy of the description there given.

In the paper referred to I stated that " the remarkable hypertrophy of the muscular coats of these vessels [namely, the arteries] seems to have for its object to assist in driving the blood onwards through the intertubular vessels in which the impediment exists."

I have long been aware that this explanation of the phenomena is physiologically untenable. It is now generally known and admitted that the minute arteries throughout the body, by the contraction of their muscular walls, have the effect of diminishing and retarding the blood-stream. The contraction of the small arteries, so far from assisting to propel the blood onwards, is, in fact, directly antagonistic to the heart's contractions, and tends to check the current of blood. The minute arteries by their contraction act as stopcocks, while by their relaxation they permit a fuller and a more rapid stream of blood, but they have no direct propelling power. The heart is the force pump which drives the blood onwards indiscriminately through the larger arterial canals. The minute arteries, by their contractile power, regulate the blood supply in accordance with the requirements of each organ and tissue. This is the doctrine which is now generally received by physiologists.

Having recently been engaged in making a careful revision of the morbid anatomy and pathology of Bright's disease in its various forms and stages, I believe that I am now in a position to give the true physiological explanation of the hypertrophy of the muscular walls of the small renal arteries, which is so constant a phenomenon in the advanced stages of all the forms of chronic Bright's kidneys. My explanation 
is this :-In proportion to the destruction of the renal glandcells, and the consequent diminution of the secretory power of the kidney, there is less demand for blood to be acted upon by the gland; the small arteries consequently contract upon their contents, so as to maintain the balance between the blood supply and the diminished secretory action of the kidney. This continued over-action of the small arteries in antagonism to the heart results in hypertrophy of their muscular walls. I believe that all the facts will be found to harmonise with this theory.

That the thickening of the walls of the small renal arteries in these cases of chronic Bright's disease is a genuine instance of hypertrophy of muscular tissue is indisputable. The two layers of fibres-an inner longitudinal, and an outer circular-retain all their characteristic appearances, and are simply increased in thickness, while the arteries, being elongated as well as thickened, often become more or less tortuous. It is equally indisputable that hypertrophy of muscular tissue implies long-continued over-action of the tissue in question. Over-action of the muscular walls of the small arteries, of necessity, diminishes the supply of blood. And it seems probable that the diminished blood supply is the result of a beneficent compensatory action. For since a kidney whose secreting structure is partially spoiled by disease is incapable of acting upon the same amount of blood as a healthy gland, it is likely that an undiminished supply of blood to a diseased kidney would tend to embarrass the organ, through engorgement of its capillary vessels, while more or less of the blood would pass through the kidney without being freed from those peculiar constituents which it is the function of the gland to secrete.

The best and purest specimens of hypertropied renal arteries are usually to be found in the advanced stages of the small granular kidney. In the large white kidneys the arteries are almost constantly found more or less hypertrophied, but in the advanced stages of the disease, more especially when the kidney has the characters of the so-called waxy or amyloid degeneration, the walls of many of the 
small arteries assume a white and wax-like appearance, and their muscular structure is thereby rendered confused and indistinct. But that this is a secondary and not a primary phenomenon is proved by the fact that, in the early stage of this waxy degeneration, while the gland is already much enlarged and presents the unmistakable features of the waxy kidney, the arteries are either unchanged or their walls are simply hypertrophied. The waxy appearance of the arteries and the Malpighian capillaries in the advanced stage of this form of Bright's disease appears to be a result of infiltration of albuminous or fibrinous material into the walls of the vessels.

In my former communication on this subject I spoke of the walls of the Malpighian capillaries as being hypertrophied. Now, although it is probable that the walls of capillaries which are long subjected to an increased pressure of blood may grow in thickness and in strength, it is nearly certain that the thickening of these Malpighian capillaries is mainly caused by the continued transudation of albumen and fibrin through their walls, and the degree of thickening appears to bear a relation to the amount of albumen in the urine during life. Virchow was the first to show that the blood-vessels in the white waxy kidneys are readily and deeply tinged by a solution of iodine. It has been abundantly proved that his application of the term amyloid to this form of degeneration was a.chemical error.

I have said that, although the muscular walls of the arteries become hypertrophied in the advanced stages of all the forms of Bright's disease yet, that the purest specimens of hypertrophy are found in the small granular kidneys. This fact would alone render it nearly certain that the hypertrophy of the arterial walls is not a result of an impeded circulation through the intertubular capillaries, for we have evidence that there is a greater impediment to the circulation through the intertubular capillaries in the large white kidney than in the small granular kidney. The amount of albumen which transudes mainly through the walls of the overgorged Malpighian capillaries affords a good index of the degree of ob- 
struction in the intertubular vessels. It is a well-known fact that the urine secreted by a large white kidney is, as a rule, much more highly charged with albumen than that secreted by the small granular kidney ; indeed, the urine in the latter form of disease is often but scantily albuminous, and sometimes entirely free from albumen. It can scarcely be doubted that one main cause of the obstructed circulation through the intertubular capillaries of the large white kidney is that the greater number of the tubes, being enlarged by intratubular growth and exudation, compress and obstruct the capillaries which lie between them. This enlargement of the tubes does not occur in the small granular kidney, except in the case of the comparatively few tubes that become dilated by accumulated secretion and thus grow into cysts. It appears, then, that there is not a direct, but rather an inverse, relation between the hypertrophy of the arterial walls and the obstruction of the intertubular capillary circulation; we infer, therefore, that the force which calls for that over-action of the renal arteries, and which results in hypertrophy of their muscular walls, has its place, not in front of these small arteries, but behind them; in short, that the arteries become hypertrophied in consequence of the resistance which they continually offer to the propulsive force of the heart.

Another fact which is inconsistent with the supposition that the thickening of the arterial walls is a consequence of an impediment in the intertubular capillaries, is that the efferent vessels of the Malpighian bodies present no appearance of thickening or dilatation. It is manifest that any obstruction to the flow of blood through the intertubular vessels would react backwards first upon the efferent vessels, and with the same force as upon the afferent arteries.

The comparative scantiness of the albumen in the urine secreted by the small granular kidneys appears to be a result partly of the absence of great obstruction in the intertubular capillaries ; partly it is due to the resistance which the wellnourished arterial walls offer to the heart's impulse.

And now, if we examine the state of the heart in these cases of chronic Bright's disease, we find that in the majority 
of cases its muscular walls, more especially those of the left ventricle, are more or less hypertrophied, even though the valves of the heart and the walls of the large arteries be healthy, and, therefore, offer no impediment to the circulation.

The frequent coexistence of cardiac hypertrophy with chronic renal disease was first pointed out by Dr. Bright, in the first volume of the 'Guy's Hospital Reports,' and he suggested two explanations- "Either that the altered quality of the blood affords irregular and unwonted stimulus to the organ immediately, or that it so affects the minute and capillary circulation as to render greater action necessary to force the blood through the distant subdivisions of the vascular system."

We now find a very remarkable phenomenon, namely, hypertrophy of muscular tissue at the two opposite extremes of the arterial system, the propulsive power of the heart and the resisting force of the arteries-at any rate, that of the renal arteries-being simultaneously increased, and, without doubt, these two antagonising hypertrophies are mutually interdependent, the one being provocative of the other. It cannot be supposed that the great hypertrophy of the left ventricle, which is often found in cases of chronic Bright's disease, is a direct result solely of the resistance offered by the renal arteries. We must look for the cause of this hypertrophy rather in the fact-that the blood, in consequence of degeneration of the kidney, being contaminated by urinary excreta and otherwise deteriorated, is impeded in its transit through the minute arteries throughout the body. We have evidence of such an impediment in the full, hard, throbbing pulse, which is a very common phenomenon in the advanced stage of chronic Bright's disease. Dr. Sanderson, in his recently published 'Handbook of the Sphygmograph' (p. 80), states that in cases of chronic Bright's disease with hypertrophy of the left ventricle, the sphygmograph affords decided evidence of increased arterial pressure. Still more conclusive evidence of an impediment to the circulation is afforded by the existence of hypertrophy of the muscular walls of the arteries in various tissues and organs. Dr. Hughlings 
Jackson lately did me the favour to bring me a kidney, some vessels from the brain, and a portion of pia mater, from a man who had died with a contracted granular kidney. There had been headache and other cerebral symptoms during life, and after death we found that, not only were the walls of the renal arteries hypertrophied, but also, though in a less degree, those of the brain and pia mater.

In another case of chronic Bright's disease, with hypertrophy of the left ventricle, I found the muscular walls of the arteries of the small intestines much hypertrophied. And more recently, in another case of the same kind, after injecting the radial artery with a blue fluid, I found very marked hypertrophy in the walls of the small subcutaneous arteries over the back of the hand.

An impeded circulation through the subcutaneous arteries affords a probable explanation of the dry and inactive state of the skin which so commonly exists in cases of chronic Bright's disease, and of the resistance offered to the action of even so powerful a diaphoretic as the hot-air bath.

Diligent and careful observation will, without doubt, soon increase the anatomical evidence of arterial resistance, to the circulation in cases of chronic Bright's disease. Action and reaction are equal, force must be met by an equivalent amount of force, and we shall probably find that the greater the hypertrophy of the left ventricle of the heart the greater and the more general is the hypertrophy of the arterial walls in various tissues and organs throughout the body. ${ }^{1}$

If I have correctly described and interpreted the structural changes in the minute arteries of the kidney, and if $I$ have given a true account of the influence exerted by the muscular walls of the small arteries upon the circulation, then it can scarcely be doubted that the same physiological principle will be found applicable to a number of pathological phenomena. The sudden alternations in the colour of the face under the influence of mental emotion can scarcely be considered pathological; yet, in passing, it may be well to refer to the phenomena as a well-known and generally admitted

VOL. I.T.

I See postscript, p. 76. 
illustration of the fact that a rapid alternation of extreme anæmia and extreme turgescence of the capillaries may result from an alternate contraction and relaxation of the small muscular arteries.

Nearly a century ago Hales ${ }^{1}$ proved, by experiments on animals recently killed, that while warm water passes very readily through the small vessels, cold water, decoction of bark, or a spirituous liquid, passes much more slowly. These experiments are explained by the principle that the small arteries, by their contraction, arrest or retard the passage of irritating or constringing liquids.

Blake $^{2}$ experimented on living animals by injecting various solutions into their blood-vessels, and he found that certain substances injected into the veins of a dog in a liquid form arrested the flow of blood through the lungs so rapidly that the animal died in less than a minute. Thus, for example, he injected fifteen grains of pure soda dissolved in six drachms of water into the jugular vein of a dog. In six seconds after the first portion of the injection was introduced the pressure in the systemic arteries began to diminish, and the mercury in the hæmadynamometer speedily sank to zero, although the pulsations of the heart still continued. In forty-five seconds the animal was dead. On opening the chest the left auricle and ventricle were found still contracting, but nearly empty, while the right cavities were so distended that their contraction was impossible; yet they too began to contract when a portion of the accumulated blood had been allowed to escape through a puncture of the heart. In another experiment he found that a hæmadynamometer in a vein indicated an increase of pressure at the same time that the arterial pressure was lessened. It is manifest, from these experiments, that paralysis of the heart was not the cause of the arrest of the circulation. In contrast with the effect of soda upon the circulation Blake found that a solution of potash, or its salts, or a solution of sulphate of magnesia, speedily arrested the heart's action. After death, thus

1 Hales' 'Statical Essays,' 1769.

: 'Edinburgh Medical and Surgical Journal,' vols. liii, liv, and lvi. 
produced, the left cavities of the heart were found filled with blood of a bright scarlet colour, affording, as he says, a certain proof that the movements of the heart had stopped before the respiration had ceased; while the only probable explanation of the effect of the soda injection is that the minute pulmonary arteries, by their contraction, arrested the passage of the contaminated blood.

In like manner, when air is forcibly blown into the vein of a living animal, or when in the human subject it is accidentally sucked in with the blood during the performance of an operation at the root of the neck or in the axilla, speedy death may result from the arrest of the frothy blood by the minute arteries of the lungs.

I have elsewhere endeavoured to prove that the arrest of blood in the minute arteries of the lungs during the collapse stage of cholera admits of but this one explanation; yet so entirely has the potent influence of the minute arteries upon the circulation been ignored by pathologists that, in their endeavour to explain the undoubted fact that the blood is impeded in its passage through the lungs of a cholera patient, they have asserted, in opposition to indisputable facts, either that the heart is paralysed or that the blood has become too thick to pass through the minute vessels.

Some of the phenomena of apnœal admit of complete explanation only by reference to the contraction of the small arteries. Thus when, in consequence of imperfection or complete suspension of the respiratory changes, unaërated black blood circulates through the systemic arteries, these arteries become distended, there is a full and throbbing pulse, and the hæmadynamometer shows an increase of pressure upon the arterial walls. The increase of pressure in the systemic arteries continues, as Dr. Reid states, for some time after the animal has ceased to struggle, and when the attempts at respiration are few and imperfect. This distension of the arterial trunks must surely be a consequence of contraction of the minute systemic arteries excited by the circulation of black blood. But when the respiratory changes

1 The term "apnœa" is here used advisedly instead of "asphyxia." 
are entirely suspended or greatly interfered with the increased pressure in the systemic arteries does not long continue, for the circulation through the lungs becomes more and more impeded and soon entirely arrested. If, when the circulation has nearly ceased, air be readmitted to the lungs the blood again instantly moves on. Upon this point $I$ extract the following from Dr. John Reid:1_- "If a tube, with a stopcock upon it, be tied into the trachea of a dog, and the stopcock turned to exclude fresh air from the lungs until the circulation of dark blood along the arteries has become much enfeebled, instantly, on the admission of fresh air into the lungs, the blood springs from the cut artery of a bright red colour, and with greatly increased force." Now, I maintain that the only probable explanation of these phenomena is that when the respiratory changes are interfered with the minute branches of the pulmonary arteries contract upon their contents and retard the blood stream, just as we have seen reason to conclude that the renal arteries keep back the blood when the secretory power of the kidney is impaired. On the other hand, as soon as the air is again freely admitted to the lungs the minute pulmonary arteries, in obedience, probably, to an influence transmitted to them through the nervous system, instantly relax and the blood passes freely onwards. In giving this explanation of the phenomena of aproea $I$ agree with Dr. Reid in the opinion that the arrest of the pulmonary circulation depends primarily upon the suspension of the respiratory changes. I differ from him in maintaining that the main and immediate cause of the arrest of blood is the contraction of the small pulmonary arteries, which must surely be more powerful and more speedy in operation than any supposed attraction between the blood and the walls of the capillaries. Dr. Reid stated that the contractility of arteries manifests itself " by slow contractions, followed by equally slow relaxations." But this statement is certainly erroneous so far as relates to the small arteries. I have before referred to facts which indicate that both contraction and relaxation of the minute muscular arteries may occur

1 'Physiological, Anatomical, and Pathological Researches,' p. 46. 
instantaneously. Mr. Lister ${ }^{1}$ has proved by numerous experiments that the arteries in the web of the frog's foot may be made to contract and to dilate with extreme rapidity. Mr. Paget $^{2}$ has demonstrated the same phenomenon in the arteries of the bat's wing. On the other hand, we have reason to believe that any retarding influence which results from an altered relation between the blood and the walls of the capillaries comes on slowly, increases very gradually, and passes away again, if it pass away at all, as slowly and as gradually as it came on.

Chronic apncea, as we all know, is invariably associated with engorgement of the pulmonary capillaries, and with more or less of watery exudation into the air-cells of the lung. In this state of chronic apncea there is evidence of capillary stasis. On the other hand, in cases of acute and rapidly fatal apnœa the minute tissue of the lung is pale and comparatively anæmic. To illustrate this condition I performed the following experiment. A dog weighing $19 \frac{1}{4} \mathrm{lbs}$. was killed by a ligature on his trachea; immediately after death the chest was opened. The right side of the heart was much distended, the auricle, in particular, standing out round and smooth, and having an elastic feel like that of an india-rubber ball distended with air. The left cavities were comparatively flaccid. A ligature having been placed round all the vessels, the heart was removed. On opening it 2 ounces of blood gushed from the distended right side, while $2 \frac{1}{2}$ drachms only escaped from the left cavities.

The lungs were pale, collapsed to an extreme degree, felt dry, and were crepitant; the right weighed $1 \frac{1}{4}$ ounce, the left an ounce and half a drachm. The pulmonary artery, before the roots of the lungs were divided, was distended; the pulmonary veins were moderately full. The venæ cavæ were distended. After cutting across the great vessels 12 ounces of blood escaped into the cavity of the chest.

Here we have evidence of an arrest of blood in its passage

1 "An Inquiry concerning the parts of the Nerrous System which regu. late the Contraction of the Arteries," 'Phil. Trans.,' 1848, p. 607.

2 'Surgical Pathology.' 
through the lungs. It is evident that the arrest did not occur in the capillaries, for these vessels were nearly bloodless ; the stoppage must have originated in the minute arteries, which by their contraction so opposed the passage of blood as to distend all the cavities and vessels leading up to the obstruction, and thus finally to arrest the circulation.

The extreme collapse of the lung when the chest is opened is a result of the extreme anæmia of its minute vessels, and the tough and non-crepitant feel of the lung is a result of the almost complete expulsion of the air from the vesicles during the extreme collapse after the chest is opened.

To illustrate the effect of post-mortem movement of the blood, another dog was killed in the same way by a ligature on the trachea, and the chest was opened after the dead body had been lying on its back for forty-four bours. The rigor mortis continued. The right cavities of the heart contained five drachms of blood; the left ventricle was firmly contracted, and contained a drachm of blood. The lungs were anæmic in their upper and anterior parts, dark and congested in the lower and back parts. Their weight was exactly the same as that of the first animal's lungs, but then it is to be observed that the first dog was a larger animal, his weight being 2 lbs. greater, and the capacity of his chest nearly twice that of the smaller dog.

In this second experiment it is possible that the distension of the right cavities of the heart had diminished after death, "the blood having made its way through various channels," as in Harvey's well-known observation on the criminal who had been executed by hanging." And, further, some blood

1 Harvey says, "I have several times opened the breast and pericardium of a man within two hours after his execution by hanging, and before the colour had totally left the face, and, in presence of many witnesses, have demonstrated the right auricle of the heart and the lungs distended with blood-the auricle, in particular, of the size of a large man's fist, and so full of blood that it looked as if it would burst. This great distension, however, had disappeared the next day, the body baving stiffened and become cold, and the blood having made its way through various channels."'Second Disquisition on the Circulation of the Blood,' Sydenham Society's Translation, p. 127. 
had gravitated into the dependent parts of the lungs, which were thus rendered dark and congested.

This experiment illustrates the importance of opening the chest as soon as possible after death, when the object is to ascertain the distribution of blood in the heart's cavities and the state of the lungs at the moment of death. This precaution is especially important in the case of patients who have died in the collapse stage of cholera.

In the 'Lancet' of November 9th, 1867, Dr. Massey, of Nottingham, describes minutely the appearances found in the body of a man four hours after his execution by hanging. "On removing the sternum and cartilages of the ribs the lungs were not to be seen, but were found to occupy a very small space at the back part of the chest, resembling the contents of a fotal thorax, the pericardial sac alone being seen. The colour of the lungs was of a darker hue than natural, especially at the bases. On cutting out the lungs a quantity of black fluid blood flowed. The structure was natural, but there was loss of crepitancy, and but very little air was contained in them. The right auricle of the heart was gorged to the greatest state of distension with blood, and the inferior cava was in the like condition; on opening the auricle a great quantity of black fluid blood gushed out. The right ventricle also contained a large amount of blood. The left auricle and ventricle were completely empty." It will be seen that the appearances described by Dr. Massey are essentially the same as those which $I$ observed in the suffocated dog.

Anæmia of the minute tissue of the lung, with engorgement of the right cavities of the heart, will be found in all cases of rapid death from apnœa-for example, in some cases of rapidly fatal apoplexy or epilepsy, cases of spasmodic croup, and cases of sudden closure of the air-passages by a foreign body, or, more rarely, by rapid croupy or diphtheritic exudation.

The same physiological principle is applicable in all these cases. The circulation through the lungs is arrested in consequence of the suspension of the function of respiration, the arrest of the circulation being a direct result of arterial 
contraction. That arterial contraction is the efficient cause of the arrest of blood is rendered still more probable by the results of some experiments to which I will briefly refer. Dr. Sanderson ${ }^{1}$ poisoned a dog with woorara, the effect of which is to suspend the respiratory movements, while the heart's contractions are for a time unaffected. Death results from apnœa, but more slowly, Dr. Sanderson found, than when apnoa is produced by plugging the trachea, and the dynamometer in a systemic artery affords no evidence of increased pressure upon the arterial walls while black blood is circulating. Bernard has shown that this poison paralyses all the motor nerves, including those of the blood-vessels. The minute arteries, both pulmonary and systemic, having thus been deprived of their contractile power, offer no impediment to the passage of the blood; the circulation, therefore, continues until the heart's contractions gradually cease. In contrast with this experiment I may refer to one which was performed by Mr. Erichsen. ${ }^{2}$ He pithed a dog in order to eliminate the disturbing element of violent struggling movements upon the arterial pressure. Then keeping up artificial respiration until he had ascertained the height of the mercury in the arterial dynamometer, he found that when the respiratory movements were suspended, and black blood consequently passed into the systemic arteries, the mercury rose to from two inches to two and a half inches higher than while respiration continued. The arterial pressure afterwards gradually diminished as the heart's action became enfeebled. In this experiment the resistance offered to the passage of black blood though the systemic arteries was clearly proved to exist after the respiratory movements had ceased and when the animal could not struggle.

The influence of the pulmonary vessels in arresting the circulation and so causing speedy death in apnoa receives indirect illustration from the curious fact, which was first discovered by Buffon, that the young of certain warmblooded animals-for example, the dog, the cat, and the

1 'Proceedings of the Royal Society,' March 7th, 1867.

2 'Edinburgh Medical and Surgical Journal,' vol. lxiii, p. 15. 
rabbit-may within a few days after their birth be immersed in water of moderate temperature for a period of sometimes half an hour before life is extinct. 1 The explanation of this interesting phenomenon is, without doubt, to be found in the fact that in the animals referred to the foramen ovale and the ductus arteriosus remain patulous for a few days after birth, so that when air is excluded from the lungs the blood passes directly from the right to the left side of the heart and to the aorta, and the circulation continues much longer than when, in older animals, the foramen ovale and the ductus arteriosus being closed, all the blood has to pass through the pulmonary vessels, and is thus subjected to their regulating and retarding influence.

And now a comparison of the phenomena of apnœa with those of renal disease appears to warrant the conclusion that an impeded circulation, the result of arterial contraction, may occur under two different conditions.

1st. When the respiratory changes in the lungs are impeded, or when the secretory action of a gland such as the kidney is impaired, the minute arteries of the lung or of the kidney-in obedience, probably, to a stimulus conveyed to them through the nerves from the capillaries-restrict and retard the blood stream. The anæmia of the pulmonary capillaries in cases of acute apnca and the overgrowth of the muscular walls of the renal arteries in cases of chronic Bright's disease are results of one and the same physiological action of the arterial stop-cocks.

2nd. The minute arteries in any part of the body may be excited to contract by their contents becoming abnormal, and therefore more or less noxious to the tissues. We have an illustration of this in the resistance which the systemic arteries offer to the passage of unaerated black blood and of blood contaminated with urinary excreta. We have another illustration of it when certain foreign materials are either accidentally in the human subject or designedly in animals

1 See Edwards, Dr. K., 'De l'Influence des Agens physiques sur la Vie,' 1824, p. 171; and Dr. J. Phillips Kay 'On Asphyxia,' 1834, p. 104, \&c. 
introduced into the veins and then arrest the flow of blood through the lungs.

We have yet another illustration of it, as I believe, in the effect of the cholera poison and its products upon the pulmonary circalation. It may be that even in this second class of cases, when contaminated blood is brought into contact with the inner surface of the arteries, the immediate incitement to the arterial contraction is a stimulus telegraphed to the arteries through the nerves from the capillaries of the tissues which are implicated.

The contraction of the minute arteries under the influence of a stimulus conveyed to them through the nerves is analogous to the contraction of the pupil which results from the stimulus of light upon the retina reflected through the motor nerves to the iris. It is also analogous to the forcible coordinate action of the respiratory muscles when there is an increased demand for air to combine with the circulating blood, or when a forcible expiratory effort is required to expel a foreign body from the air-passages.

The arrest of morbid blood in its passage through the vessels is probably the result of a law or principle which, in general, is as wholesome as the arrest of a foreign body in its passage through the larynx, yet in particular cases the arterial or the laryngeal spasm by which this generally wholesome arrest is effected or attempted may be itself the immediate cause of death.

The late Dr. Alison, and many pathologists since Dr. Alison, have taught that there exists in the minute bloodvessels a power by which, in various morbid states of the tissues and of the blood, the heart's action is antagonised, and the circulation of the blood is impeded; but it is remarkable that this retarding influence has been almost universally believed to exist in the capillaries which have no contractile power, while the stop-cock action of the small arteries, with their contractile, muscular walls, which physiologists now systematically teach, has been almost entirely overlooked and ignored by pathologists. One of the main objects of this communication is to direct attention to this 
action of the small arteries in varions pathological states of the system, and to afford anatomical as well as physiological evidence of its reality and its powerful influence. Henceforth, when we are investigating the subject of capillary engorgement and stasis, we shall have to inquire whether even this is not, in part at least, attributable to the diminished vis à tergo which must result from contraction of the minute arteries. To attribute capillary obstruction and stasis to some mysterious attraction or want of attraction between the blood and the minute vessels, while the action of the arterial sphincters is disregarded, is an inconsistency which would have its counterpart in a fanciful attempt to explain the retention of urine in the bladder by a supposed attraction or want of attraction between the urethra and the urine, while the action of the sphincter vesica is taken for nought.

As exceptions to the general disregard by pathologists of the influence of the small arteries upon the circulation, it is right to mention that Mr. Erichsen, in his very able and interesting article on asphyxia, published in 1845 ('Edinburgh Medical and Surgical Journal,' vol. lxiii), attributes the impeded transit of unaërated black blood through the systemic vessels to the contraction of the small arteries upon their abnormal contents. And Kussmaul and Tenner, in a monograph translated by the New Sydenham Society, having proved that anæmia of the brain is a common cause of epileptiform convulsions, refer to a number of facts which render it highly probable that in many cases of epilepsy the anæmia of the brain, which is the immediate cause of the convulsive paroxysm, is a result of a sudden contraction of the muscular walls of the minute cerebral arteries. This theory of arterial contraction as the immediate cause of cerebral anæmia in some cases of epilepsy has been adopted by Dr. BrownSéquard, by Dr. Russell Reynolds, and by other pathologists. Lastly, the sphygmographic observations of Marey and his followers are based upon the principle that, while the blood is driven onwards by the heart, the minute arteries, by the contraction of their muscular walls, antagonise the heart and regulate the blood supply to the various tissues and organs. 
Postscript.-Since this paper was read we have found at King's College Hospital that in every fatal case of chronic Bright's disease with hypertrophy of the left ventricle there has been decided hypertrophy of the arterial walls in most of the tissues examined, not only in the kidneys, but also in the skin, the intestines, the muscles, and the pia mater. About ten cases have been examined. In some instances the kidneys have been small and granular, in others large, smooth, and pale; the hypertrophy of the arterial walls in the various tissues has been found in both classes of cases.

The facts hitherto observed all point to the conclusion that hypertrophy of the walls of the small arteries is a result of their continued overaction in opposition to the heart. Mere distension of the arteries in consequence of an obstruction in front does not cause hypertrophy of their walls. This is proved by specimens showing no thickening of the minute pulmonary arteries in emphysematous lungs, where there has been extensive obliteration of pulmonary capillaries, with hypertrophy of the right ventricle; and by other specimens showing no thickening of the arteries in the lung of a patient who had chronic incompetence of both the mitral and aortic valves, with much pulmonary engorgement and hæmoptysis. The arteries are best seen after injection with Prussian blue.

Dr. Kelly, the able pathologist of the hospital, has bestowed much time and labour upon the details of this interesting subject, and he will shortly publish the results.

I am indebted to my friend Dr. Hyde Salter for the accurate drawings of the arteries, both normal and hypertrophied, with which this paper is illustrated. 


\section{DESCRIPTION OF THE PLATES.}

The arteries in all the specimens have their canals filled with Prussian blue. The magnifying power is 200 diameters in each case, except Plate II, Fig. 1, which is drawn from a specimen magnified 100 diameters.

\section{Plate I.}

Fig. 1.-Normal artery from the pia mater.

Fig. 2.-Arteries with hypertrophied walls from the pia mater.

Fig. 3.-Artery with hypertrophied walls from the small intestines.

\section{Plate II.}

Fig. 1.-Normal artery from the skin.

Fig. 2.-Artery with hypertrophied walls from the skin. It will be seen that the walls of the comparatively large artery (Plate II, fig. 1) have the same structure and about the same thickness as the walls of the smallest branches of the artery in this figure.

Fig. 3.-Artery with hypertrophied walls from muscle.

Fig. 4.-Artery with hypertrophied walls from the kidney. In the hypertrophied renal arteries there is always an inner longitudinal layer of fibres about equal in thickness to the outer circular layer. This thickened longitudinal layer has not been seen in the hypertrophied arteries from any other tissue.

N.B.-All the hypertrophied arteries are from the subjects of chronic Bright's disease. 


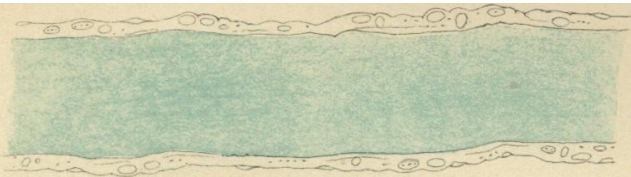

Fig 1.
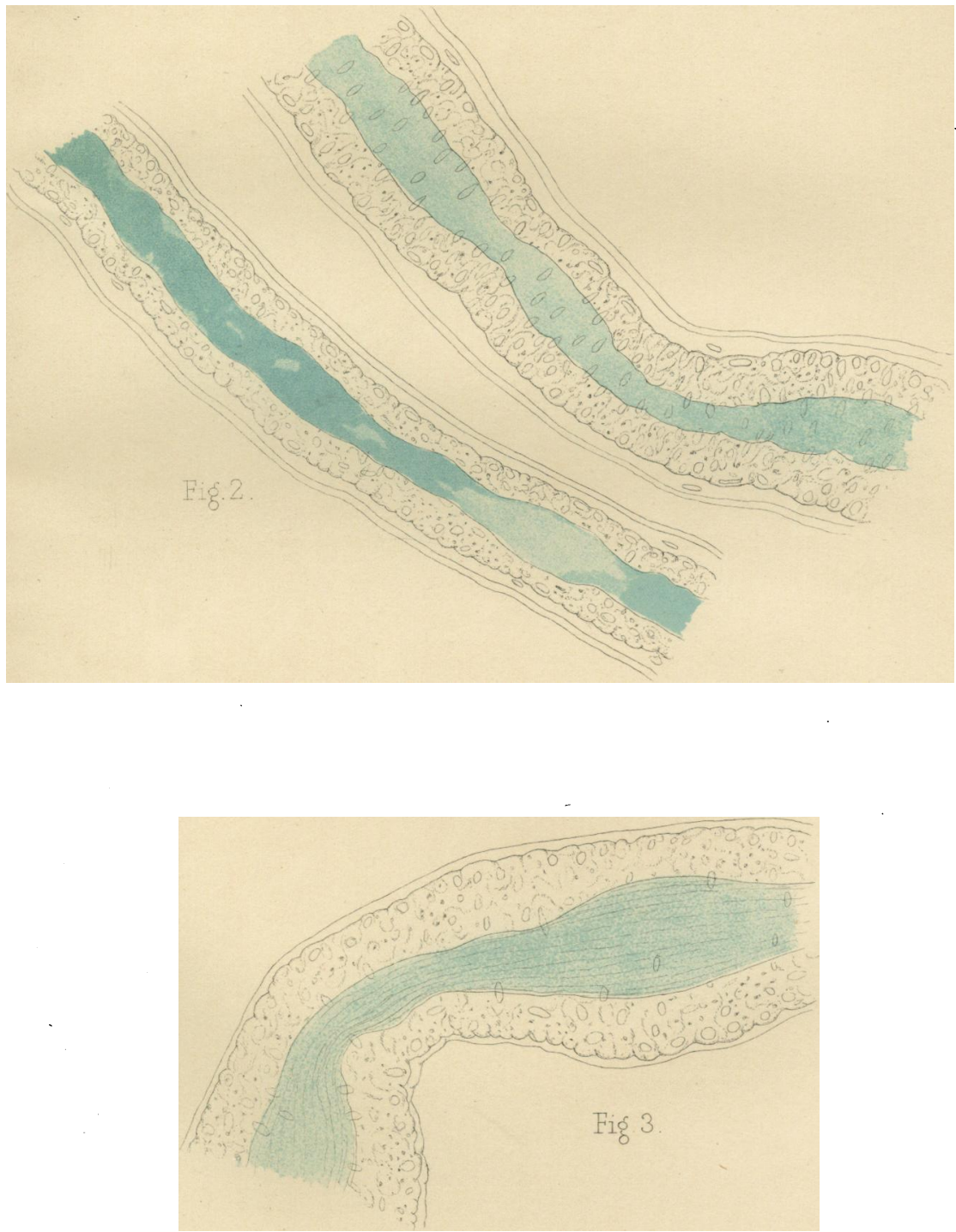

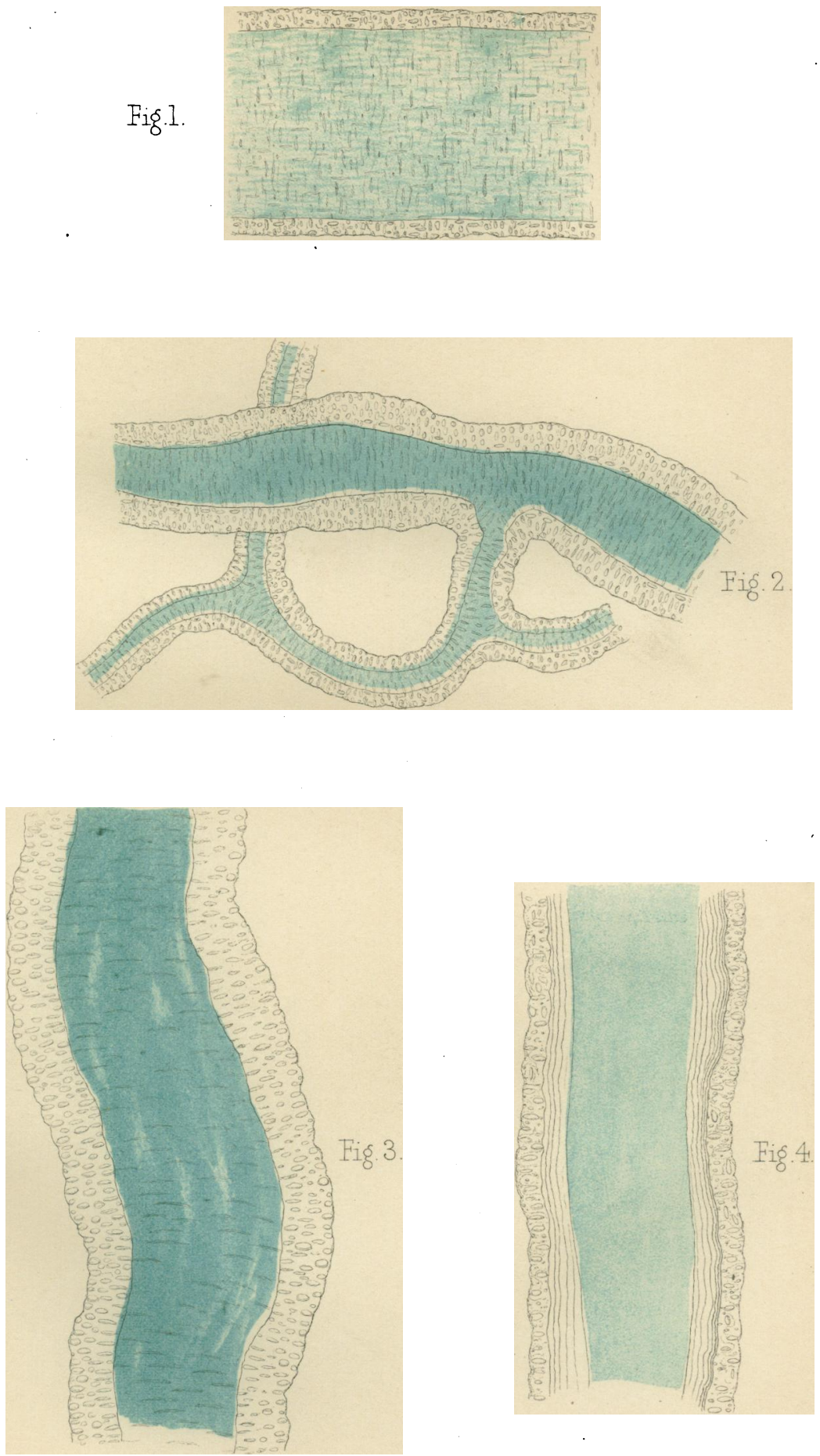